\title{
ANÁLISE DO PREENCHIMENTO DE DECLARAÇÕES DE ÓBITOS EM LOCALIDADE DO ESTADO DO-RIO GRANDE DO SUL (BRASIL), 1987
}

\author{
Irajá Carneiro Heckmann* \\ Luis Henrique Canani** \\ Urbano Leonel Sant'anna* \\ Ronaldo Bordin***
}

\begin{abstract}
HECKMANN, I.C. et al. Análise do preenchimento de declaraçðes de óbitos en localidade do Estado do Rio Grande do Sul (Brasil). 1987. Rev. Saúde públ., S. Paulo, 23:292-7, 1989.

RESUMO: É analisado o preenchimento dos ítens existentes nas declaraçōes de óbito registradas para o Município de Viamão, Rio Grande do Sul, Brasil, durante 1987. Os ítens foram sistematizados segundo as informaçðes em branco, ignoradas ou incorretas. Observou-se uma pequena omissão no preenchimento dos dados de identificação do falecido, sendo maior no tocante aos antecedentes sociais e obstétricos, assistência médica e casos de morte violenta. Para melhorar o preenchimento das declarações de óbito sugere-se processo de sensibilização contínua do médico para a importância dos dados por ele assinalados, capacitação de funcionários administrativos que lidam com as declarações e realização de estudos de mortalidade setorizados por serviços de saúde.
\end{abstract}

DESCRITORES: Atestados de óbitos. Controle de formulários e registros. Mortalidade.

\section{INTRODUÇĀO}

Poucos são os estudos realizados no Brasil que enfocam a qualidade das informações registradas nas declarações de óbito. Apesar de datar de 1948, o modelo internacional proposto pela Organização Mundial de Saúde ${ }^{2}$ e de desde 1976 ser utilizado no Brasil uma declaração de óbito padronizada 7,9 , estudos nesta área mostram não ser de boa qualidade os registros contidos nas declarações $2,5,8,10$.

Pereira e Castro ${ }^{8}$, ao avaliarem o preenchimento de declarações de óbito de Brasília (1977-78), encontraram percentagem reduzida de dados desconhecidos quanto à identificação do falecido, mas com não preenchimento elevado de dados referentes à gravidez e ao parto (de até $50 \%$ dos óbitos fetais e $67 \%$ dos menores de um ano), assistência médica e casos de morte violenta.

Explicação para tal situação estaria no fato de os curriculos médicos nem sempre enfatizarem a importância do correto preenchimento das declaraçōes de óbito. Não sendo incomum, neste contexto, o médico deparar-se pela primeira vez com a declaração de óbito no momento de preenchê-la, enquanto exigência legal ao sepultamento, perdendo a perspectiva da importância das informaçőes por ele assinaladas para o planejamento das ações de saúde ${ }^{3}$. Fato este já reconhecido há séculos, sendo este documento uma fonte estatística vital e base da moderna epidemiologia ${ }^{4}$.

O objetivo deste estudo é apresentar uma avaliação do preenchimento dos itens da declaração de óbito diretamente ligados à saúde pública, quais sejam, a parte II (identificação do falecido), parte III (para menores de um ano ou óbito fetal), parte IV (causa da morte) e parte V (em caso de morte violenta). Desta forma, embora utilizando de declaraçōes originais, seguiu-se modelo anteriormente apresentado por Pereira e Castro ${ }^{8}$ e restringindo-se aos óbitos registrados para o Município de Viamão (RS), durante o ano de 1987.

\section{MATERIAL E MÉTODOS}

O estudo consiste na análise das declarações de óbito das pessoas que apresentaram residência habitual no Município de Viamão, integrante da região metropolitana de Porto Alegre (RS), em número de 887 para o ano de 1987.

A consulta das declarações de óbito se deu

* Acadêmicos da Faculdade de Medicina da Universidade Federal do Rio Grande do Sul (Travessa Farroupilha, 95 90420 - Porto Alegre, RS - Brasil).

** Assessoria Científica da Faculdade de Medicina da Universidade Federal do Rio Grande do Sul - Rua Ramiro Barcelos, 2.600 - 90210 - Porto Alegre, RS - Brasil.

*** Departamento de Medicina Preventiva da Faculdade de Medicina da Universidade Federal do Rio Grande do Sul e da Assessoria Científica da Faculdade de Medicina da Universidade Federal do Rio Grande do Sul - Rua Ramiro Barcelos, 2.600 - 90210 - Porto Alegre, RS - Brasil. 
nos arquivos da Secretaria da Saúde e Meio Ambiente do Estado do Rio Grande do Sul (SSMA/RS), que recebe uma das vias de cada declaração dos cartórios. A classificação dos ítens da declaração é feita segundo o "Manual de Instruçð̃es para o Preenchimento de Declaração de Óbito"', ao passo que a codificação das classes incluídas nesses ítens se realiza segundo o "Manual de Instruç̃es da Planilha para a Codificação da Declaração de Óbito", segundo a Classificação Internacional de Doenças ${ }^{1}$, para a causa básica de óbito.

No presente trabalho quantificou-se para cada item das partes II a $\mathbf{V}$ da declaração de óbito o número e percentual de informações em branco (situação na qual o médito não assinalou nenhuma resposta), ignorada (situação na qual se tentou obter a informação, não havendo sucesso) e incorreta (quando ocorreu o preenchimento de duas opçóes ou que o dado preenchido não permitia informação, levando-se em conta outros dados da declaração). Cabe assinalar que um item pode ser considerado correto mesmo quando foi preenchido com a palavra ignorado.

Todos os resultados foram divididos em três classes de óbito: fetal, de menores de um ano e com idade igual ou superior a um ano de idade.

\section{RESULTADOS}

Durante 1987 foram registrados 887 óbitos no Município de Viamão (RS), sendo $54(6,1 \%)$ mortes fetais, $76(8,6 \%)$ falecimentos de menores de um ano de idade e $757(85,3 \%)$ de maiores de um ano.

$\mathrm{Na}$ Tabela 1 estão enumeradas as informações em branco e as ignoradas contidas na parte II da declaração de óbito, referente aos dados de identificação do falecido ou sobre o óbito fetal, filiação. Nesta parte da declaração ocorreram preenchimentos incorretos nos óbitos fetais apenas em duas $(3,7 \%)$ declaraçồes no item 3 (óbito fetal). Nos óbitos verificados em menores de um ano ocorreram incorreções nos itens 3 e 17 , na ordem de $3(3,9 \%)$ e $17(22,4 \%)$, respectivamente. Já quanto às incorreções em óbitos em maiores de um ano, ocorreram $2(0,3 \%)$ incorreçסes no item $3,3(0,4 \%)$ no item 6 (estado civil), $217(28,7 \%)$ no item 16 (ocupação habitual), $153(20,2 \%)$ no item 17 (naturalidade) e uma $(0,1 \%)$ no item 18 (grau de instrução).

Os preenchimentos dos dados de óbitos em menores de um ano ou obito fetal, incluindo as informaçōes referentes às condiçðes de vida familiar, de gestações da mãe, condições de morte e peso ao nascer do falecido, que compóem a parte III da declaração de óbito, encontram-se na Tabela 2. Não foram encontrados preenchimentos incorretos nesta parte da declaração, com apenas uma informação ignorada $(1,3 \%)$ em menores de um ano, no item 21 (ocupação habitual do pai).

Os dados referentes à assistência médica, parte IV da declaração de óbito, estão expressos na Tabela 3. Chama especial atenção o número de campos em branco no item "intervalo entre o início da doença e a morte", atingindo em alguns casos $98,7 \%$. O item "causa de morte" não foi estudado neste trabalho, embora o mesmo não tenha apresentado omissão quanto ao seu preenchimento em nenhum atestado, ao menos de forma parcial. Não foram encontrados preenchimentos incorretos nesta parte da declaração.

Os ítens não preenchidos e ignorados no tocante às informaçðes sobre morte violenta (parte $\mathrm{V}$ da declaração) estão na Tabela 4. Ocorreram 68 mortes violentas durante 1987 neste $\mathrm{Mu}$ nicípio, perfazendo $7,7 \%$ do total de 6 bitos registrados. Embora não tenham sido encontrados preenchimentos incorretos, a omissão de registro quanto a acidentes de trabalho e local de ocorrência dos mesmos, os menos preenchidos desta parte da declaração, manteve-se acima de $80 \%$.

\section{DISCUSSĀO E CONCLUSÃO}

Os resultados encontrados demonstram omissão de preenchimento dos itens de identificação do falecido (Tabela 1), em média inferior a $10 \%$, sofrendo um incremento importante no tocante aos antecedentes sociais e obstétricos (Tabela 2), assistência médica (Tabela 3) e na parte destinada a registros por morte violenta, notadamente se acidente de trabalho e local (Tabela 4), referendando dados anteriormente encontrados por Pereira e Castro ${ }^{8}$, para o $\mathrm{Mu}$ nicípio de Brasília, DF.

A baixa omissão de preenchimento dos dados de identificação, se comparado aos demais, pode ser atribuído ao rápido registro dos mesmos por parte do médico, sem maiores problemas de obtenção das informaçð̃es perante os familiares do falecido, e também ao fato de, em o óbito ocorrendo em instituições de saúde, tal campo ser preenchido por pessoal administrativo. Entretanto, deve ser observado o número de ítens em branco em maiores de um ano que, acrescido de um índice de $2,8 \%$ de preenchimentos incorretos da ocupação habitual do falecido, delimita um item costumeiramente desprezado para informação. Isso decorre devido ao registro 


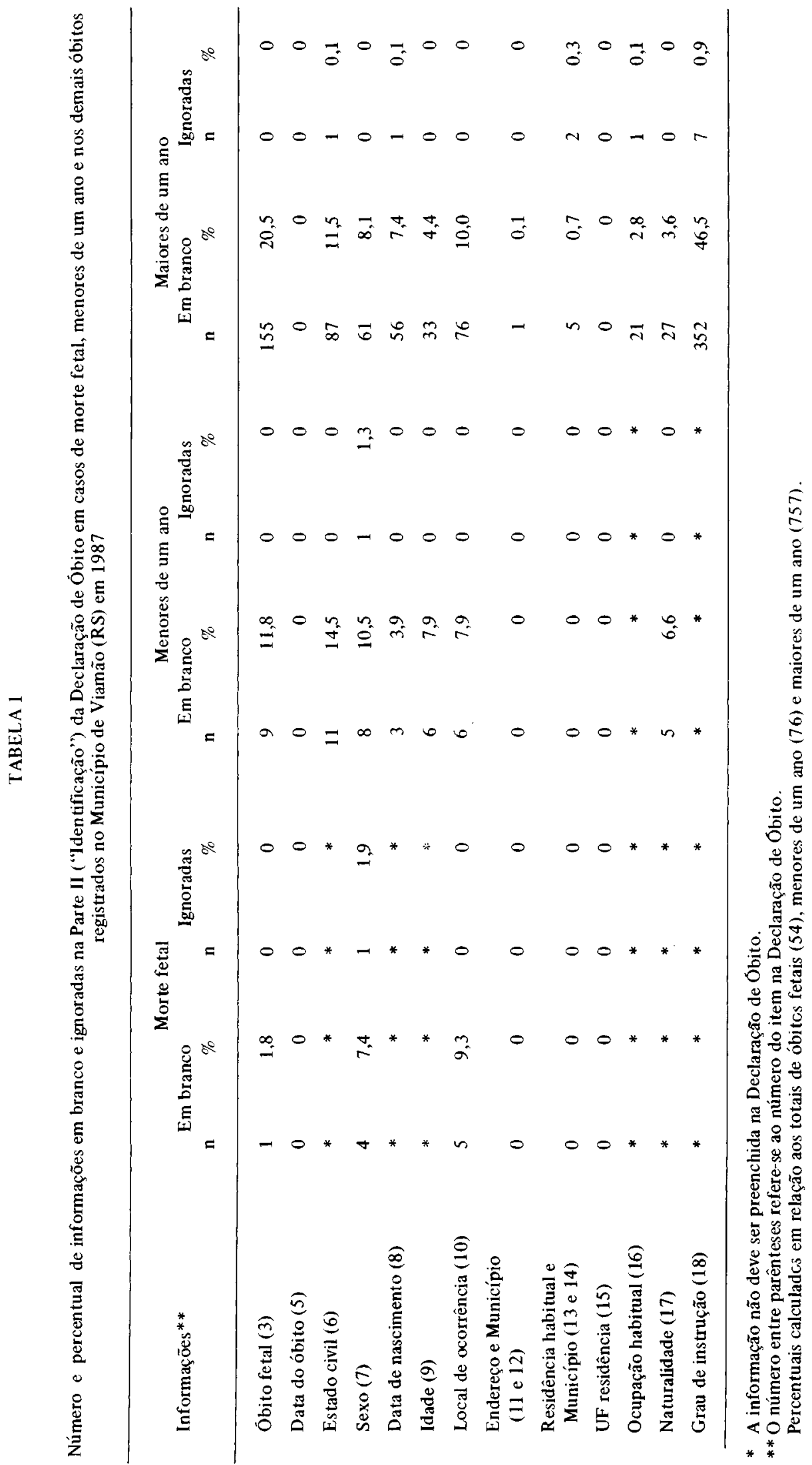


TABELA 2

Número e percentual de informaçōes em branco na Parte III ("Menor de um ano ou óbito fetal") da Declaração de Óbito, registrados no Município de Viamão (RS) em 1987.

\begin{tabular}{|c|c|c|c|c|}
\hline \multirow{2}{*}{ Informaçōes* } & \multicolumn{2}{|c|}{ Fetais } & \multicolumn{2}{|c|}{ Menor um ano } \\
\hline & $\mathbf{n}$ & $\%$ & $\mathrm{n}$ & $\%$ \\
\hline \multicolumn{5}{|l|}{ Pai: } \\
\hline Ocupação habitual (21) & 12 & 22,2 & 19 & 25,0 \\
\hline Grau de instrução (22) & 42 & 77,8 & 58 & 76,3 \\
\hline \multicolumn{5}{|l|}{ Mãe: } \\
\hline Ocupação habitual (23) & 6 & 11,1 & 8 & 10,5 \\
\hline Idade $(24)$ & 7 & 13,0 & 37 & 48,7 \\
\hline Grau de instrução (25) & 36 & 66,7 & 48 & 63,2 \\
\hline \multicolumn{5}{|l|}{ Gravidez e parto: } \\
\hline No de filhos vivos (26) & 38 & 70,4 & 63 & 82,9 \\
\hline No de filhos mortos $(26)$ & 38 & 70,4 & 62 & 81,6 \\
\hline Duração da gestação (27) & 30 & 55,6 & 60 & 78,9 \\
\hline Gravidez (28) & 33 & 66,1 & 58 & 76,3 \\
\hline Tipo de parto (29) & 25 & 46,3 & 60 & 78,9 \\
\hline Morte do feto (parto) (30) & 25 & 46,3 & 68 & 89,5 \\
\hline Peso ao nascer ( 31$)$ & 23 & 42,6 & 21 & 27,6 \\
\hline
\end{tabular}

* O número entre parênteses refere-se ao número do item da Declaração de Obito.

Percentuais calculados em relação aos totais de óbitos fetais (54) e de menores de um ano (76).

de ocupações vagas, tais como aposentado, funcionário público e outras que não permitem uma adequada classificação. Nesta parte da declaração também deve ser salientado que em quase metade das declaraçōes de óbito em maiores de um ano de idade não consta o seu grau de instrução.

No tocante aos antecedentes sociais e obstétricos, a ser preenchido obrigatoriamente nos casos de óbito em menores de um ano ou fetal, de suma importância na análise das influências destes fatores no produto da concepção, observou-se uma omissão de registro de mais de $2 / 3$ das declarações em menores de um ano dos ítens referentes à gravidez e parto, com percen- tual menor nos óbitos fetais, embora importante (variando de 42 a $70 \%$ ).

A parte IV da declaração de óbito é de responsabilidade exclusiva do médico e fornece dados referentes à assistência médica e ds causas de morte. A Tabela 3 delimita o grau de omissão de preenchimento do item "intervalo entre o surgimento da doença e a morte" como de no mínimo $87,5 \%$, em maiores de um ano, chegando a $98 \%$ em óbitos fetais e menores de um ano. Tais índices apresentam a mesma magnitude em estudos realizados em São Paulo,s.

Os itens referentes à parte $\mathrm{V}$ da declaração são somente assinalados em caso de morte por homicídio, suicídio ou acidente e naqueles em

\section{TABELA 3}

Número e percentual de informaçōes em branco e ignoradas da Parte IV ("A testado médico") da Declaração de Óbito em casos de morte fetal, menores de um ano e nos demais óbitos registrados no Município de Viamão (RS) em 1987.

\begin{tabular}{|c|c|c|c|c|c|c|c|c|c|c|c|c|}
\hline \multirow{3}{*}{ Informaçōes * } & \multicolumn{4}{|c|}{ Morte fetal } & \multicolumn{4}{|c|}{ Menores de um ano } & \multicolumn{4}{|c|}{ Maiores de um ano } \\
\hline & \multicolumn{2}{|c|}{ Em branco } & \multicolumn{2}{|c|}{ Ignorada } & \multicolumn{2}{|c|}{ Em branco } & \multicolumn{2}{|c|}{ Ignorada } & \multicolumn{2}{|c|}{ Em branco } & \multicolumn{2}{|c|}{ Ignorada } \\
\hline & $\mathrm{n}$ & $\%$ & $\mathbf{n}$ & $\%$ & $\mathrm{n}$ & $\%$ & $\mathrm{n}$ & $\%$ & $\mathrm{n}$ & $\%$ & $\mathrm{n}$ & $\%$ \\
\hline Assistência médica (32) & 29 & 53,7 & 3 & 5,6 & 38 & 50,0 & 0 & 0 & 265 & 35,0 & 2 & 0,3 \\
\hline Médico (33) & 29 & 53,7 & 0 & 0 & 32 & 42,1 & 0 & 0 & 212 & 28,0 & 0 & 0 \\
\hline Diagnóstico (34) & 31 & 57,4 & 0 & 0 & 31 & 40,8 & 0 & 0 & 227 & 30,0 & 0 & 0 \\
\hline Intervalo entre a doença & & & & & & & & & & & & \\
\hline e a morte $(35)$ & 53 & 98,1 & 0 & 0 & 75 & 98,7 & 0 & 0 & 662 & 87,4 & 1 & 0,1 \\
\hline
\end{tabular}

* O número entre parênteses refere-se ao número do item da Declaração de Óbito.

Percentuais calculados em relação aos totais de óbitos fetais (54), em menores de um ano (76) e maiores de um ano de idade (757). 
TABELA 4

Número e percentual de informações em branco e ignoradas da Parte V ("Violência") da Declaração de Óbito em menores e maiores de um ano de idade, registrados no Município de Viamão (RS) em 1987.

\begin{tabular}{|c|c|c|c|c|c|c|c|c|}
\hline \multirow{3}{*}{ Informaçāo* } & \multicolumn{4}{|c|}{ Menores de um ano } & \multicolumn{4}{|c|}{ Maiores de um ano } \\
\hline & \multicolumn{2}{|c|}{ Em branco } & \multicolumn{2}{|c|}{ Ignorada } & \multicolumn{2}{|c|}{ Em branco } & \multicolumn{2}{|c|}{ Ignorada } \\
\hline & $\mathrm{n}$ & $\%$ & $\mathrm{n}$ & $\%$ & $\mathrm{n}$ & $\%$ & $\mathrm{n}$ & $\%$ \\
\hline Tipo de violência (37) & 0 & 0 & 0 & 0 & 33 & 49,2 & 4 & 6,0 \\
\hline Acidente de trabalho (38) & 1 & 100,0 & 0 & 0 & 65 & 97,0 & 1 & 1,5 \\
\hline Local do acidente (39) & 0 & 0 & 0 & 0 & 55 & 82,1 & 1 & 1,5 \\
\hline
\end{tabular}

* O número entre parênteses refere-se ao número do item da Declaração de Óbito.

Percentuais calculados em relação ao total de mortes violentas em menores de um ano (1) e maiores de um ano de idade (67).

que a morte é evidentemente violenta, isto é, não natural, mas que o tipo de violência é desconhecido. Este item não foi preenchido em $46 \%$ dos casos e os quanto a acidentes de trabalho, em $82 \%$, contribuindo sobremaneira para a manutenção da elevada taxa de subnotificação de acidentes de trabalho no Brasil.

Levando em consideração os dados acima, os autores concluem que embora o Brasil apresente um modelo padrão de declaração de óbito há mais de dez anos, bem como um manual para instruir o seu preenchimento, não se verificou um bom registro de informações neste Múnicípio estudado deste importante instrumento de obtenção de dados para a descrição das características de mortalidade da região. Neste sentido, os autores apontam como possiveis formas de minimizar estas omissões ou incorreçס̃es de preenchimento:

a) uma contínua sensibilização do médico para a importância dos dados contidos nas declaraçōes de obito no planejamento das medidas de saúde pública. Tal sensibilização pode ser obtida através de uma maior atenção à declaração nos cursos médicos e da devolução sistemática dos dados assinalados (por médico, por serviço de saúde, por região geográfica ou forma a ser otimizada pelas unidades de informática), no intuito do médico conseguir visualizar de forma direta sua participação nos dados agregados que ocasionalmente toma conhecimento;

b) identificação e capacitação dos funcionários administrativos que comumente preenchem alguma parte da declaração; e

c) realização de estudos de mortalidade por serviço de saúde, mobilizando o corpo clínico para sua realização e análise. Tais estudos são de fácil apreensão metodológica e apresentam um custo reduzido para sua realização, sendo de indiscutivel valor para que o serviço discuta a qualidade do preenchimento das declarações de óbito, provavelmente alcançando amplitude superior de conscientização quanto à necessidade de um registro mais correto do que as duas medidas acima enumeradas.

\section{AGRADECIMENTOS}

Ao Sr. Riograndino Carvalho Borges, funcionário da Secretaria da Saúde e Meio Ambiente do Estado do Rio Grande do Sul, pela colaboração na obtenção dos dados.

HECKMANN, I.C. et al. [An analysis of death certificate information in a locality of the State of Rio Grande do Sul (Brazil), 1987]. Rev. Saúde públ., S. Paulo, 23:292-7, 1989.

\footnotetext{
ABSTRACT: It was analyzed the quality of death certificate information for deaths registed in Viamão county (Rio Grande do Sul, Brazil) during 1987, considering unfilled blanks, and unknown and incorrect responses. Small omissions were found in data identifying the person who had died; larger omissions in data concerning social and obstetric history, medical assistance, and violent deaths. To improve the quality of death certificate information it was suggested that physicians be continually reminded of the importance of giving the correct information on death certificates to allow for'their later adequate administrative and research use.
}

KEYWORDS: Death certificates. Forms and records control. Mortality. 


\section{REFERÊNCIAS BIBLIOGRÁFICAS}

1. CENTRO DA ORGANIZAÇĀO MUNDIAL DA SAÚDE PARA CLASSIFICAÇ̧̃̃O DE DOENÇAS EM PORTUGUÊS. Manual de classificação estatística internacional de doenças, lesões e causas de óbito. São Paulo, 1980.

2. FONSECA, L.A.M. \& LAURENTI, R. A qualidade da certificação médica da causa de morte em São Paulo. Rev. Saúde públ., S. Paulo, 8:21-9, 1974.

3. LAURENTI, R. \& JORGE, M.H.P. O atestado de óbito. 2a ed., São Paulo, Centro da OMS para a Classificação de Doenças em Português, 1987.

4. McMAHON, B. \& PUGH, T.F. Epidemiology: principles and methods. Boston, Little Brow and Co., 1970.

5. MILANESI, M.L. \& LAURENTI, R. O estudo interamericano de mortalidade em São Paulo. I - Estado geral da certificação médica da causa de óbito no distrito da capital. Rev. Ass. med. bras., 10: 111-6, 1964.

6. MINISTÉRIO DA SAÚDE. Manual de instruçōes para preenchimento de declaração de óbito. 2a ed. Brasília, 1985. (Série A: Normas e Manuais Técnicos, 24).
7. MINISTÉRIO DA SAÚDE. Sistema de Informações de Saúde. Sub-sistema de Informações sobre Mortalidade. Manual de procedimentos e operações. Brasília, s.d.

8. PEREIRA, M.G. \& CASTRO, E.S. Avaliação do preenchimento de declarações de óbitos: Brasília, DF (Brasil), 1977-1.978. Rev. Saúde públ., S. Paulo, 15:14-9, 1981.

9. REUNIĀO NACIONAL SOBRE SISTEMA DE INFORMAÇĀO DE SAÚDE, 1․, Brasília, 1975. Brasília, Ministério da Saúde, 1975.

10. SILVEIRA, M.H. \& LAURENTI, R. Os eventos vitais: aspectos de seus registros e interrelação da legislação vigente com as estatísticas de saúde. Rev. Saúde públ., S. Paulo, 7:37-50, 1973.

Recebido para publicação em 10/1/1989. Reapresentado em 24/5/1989.

Aprovado para publicação em 16/6/1989. 